\title{
Things to be Know about the Coronavirus Illness (The Era of Covid-19)
}

\section{Vinayaka AM*}

Department of Periodontics and Implantology, Karnataka, India

*Corresponding Author: Vinayaka AM, Department of Periodontics and

Implantology, Karnataka, India.
Received: March 20, 2020

Published: April 10, 2020

(C) All rights are reserved by Vinayaka AM.
Coronavirus illness 2019 (COVID-19) is an infectious disease caused by the virus strain "severe acute metabolic process syndrome coronavirus 2" (SARS-CoV-2). The illness was $1^{\text {st }}$ known in 2019 in Wuhan, China, and has since unfold globally, leading to the 2019-20 coronavirus pandemic. Common symptoms include fever, cough and shortness of breath. Muscle pain, sputum production and raw throat area unit less common. While the bulk of cases end in gentle symptoms, some attain severe pneumonia and multi-organ failure. As of twenty March 2020, the rate of deaths per range of diagnosed cases is four. $1 \%$, however ranges from zero. $2 \%$ to fifteen betting on age and different health issues [1,2].

The infection is often unfold from one person to a different via respiratory droplets produced throughout coughing and sneeze. Time from exposure to onset of symptoms is usually between two and fourteen days, with a mean of five days. The normal methodology of designation is by reverse transcription enzyme chain reaction (rRT-PCR) from a nasopharyngeal swab. The infection can even be diagnosed from a mixture of symptoms, risk factors and a chest CT scan showing options of respiratory disease [2,3].

Recommended measures to forestall infection embrace frequent hand laundry, maintaining distance from others (social distancing) and keeping hands off from the face. The use of masks is usually recommended for those that suspect they need the virus and their caregivers, however not the overall public. There is no vaccine or specific antiviral treatment for COVID-19. Management involves treatment of symptoms, confirming care, isolation, and experimental measures [3].

The World Health Organization (WHO) proclaimed the 2019-20 coronavirus flare-up a pandemic and a Public Health Emergency of International Concern (PHEIC). Evidence of nearby transmission of the sickness has been found in numerous nations over each of the six WHO regions [1].

Signs and symptoms

In spite of the fact that those contaminated with the infection might be asymptomatic, many create influenza like indications including fever, hack and brevity of breath. Less usually, upper respiratory side effects, for example, sniffling, runny nose, or sore throat might be seen. Gastrointestinal manifestations, for example, sickness, heaving, and loose bowels are found in a minority of cases and a portion of the underlying cases in China gave just heart side effects, similar to chest snugness and palpitations. In a few, the ailment may advance to pneumonia, multi-organ disappointment, and death $[4,5]$.

As is basic with diseases, there is a deferral from when an individual is tainted with the infection to when they create indications, known as the hatching time frame. The hatching time frame for COVID-19 is commonly five to six days yet may go from two to fourteen days [5].

\section{Causes}

The disease is as a result of the virus intense acute respiration syndrome coronavirus 2 (SARS-CoV-2), previously referred to as the 2019 novel coronavirus (2019-nCoV). It is frequently unfold between human beings via respiration droplets from coughs and sneezes. The virus can remain feasible for up to 3 days on plastic and stainless steel. SARS-CoV-2 can last as long as three days, or in aerosols for three hours. The virus has additionally been found in faeces, but as of March 2020 it is unknown whether transmission via faeces is possible, and the hazard is expected to be low $[6,7]$.

The lungs are the organs most tormented by COVID-19 due to the fact the virus accesses host cells through the enzyme ACE2, which is most abundant in the kind II alveolar cells of the lungs. The virus makes use of a unique surface glycoprotein, called "spike", to hook up with ACE2 and enter the host cell. The density of ACE2 in every tissue correlates with the severity of the sickness in that tissue and some have suggested that decreasing ACE2 activity is probably shielding, although any other view is that growing ACE2 the usage of Angiotensin II receptor blocker medications will be protecting and that those hypotheses need to be tested. As the alveolar sickness progresses, respiratory failure would possibly expand and death might also follow [7].

The virus is idea to be herbal and feature an animal origin, via spillover infection. It changed into first transmitted to human be- 
ings in Wuhan, China, in November or December 2019, and the number one source of infection have become human-to-human transmission by using early January 2020. The earliest known infection passed off on Revolutionary Organization 17 November 2019. As of 14 March 2020, 67,790 instances and 3,0.5 deaths due to the virus had been said in Hubei province; a case fatality rate (CFR) of $4.54 \%$ [8].

\section{Diagnosis}

The WHO has distributed a few testing conventions for the disease. The standard strategy for testing is continuous converse interpretation polymerase chain response (rRT-PCR). The test should be possible on respiratory examples got by different strategies, including a nasopharyngeal swab or sputum sample. Results are commonly accessible inside a couple of hours to two days. Blood tests can be utilized, yet these require two blood tests dismantled two weeks and the outcomes have minimal prompt value. Chinese researchers had the option to disengage a strain of the coronavirus and distribute the hereditary arrangement with the goal that labs over the world could freely create polymerase chain response (PCR) tests to distinguish contamination by the virus [9].

Starting at 26 February 2020, there were no immune response tests or purpose of-care tests however endeavors to create them are ongoing. Symptomatic rules discharged by Zhongnan Hospital of Wuhan University proposed techniques for distinguishing diseases dependent on clinical highlights and epidemiological hazard. These included recognizing individuals who had in any event two of the accompanying indications notwithstanding a background marked by movement to Wuhan or contact with other contaminated individuals: fever, imaging highlights of pneumonia, typical or decreased white platelet check, or diminished lymphocyte count. An investigation distributed by a group at the Tongji Hospital in Wuhan on 26 February 2020 demonstrated that a chest CT examine for COVID-19 has more prominent affectability (98\%) than the polymerase chain response $(71 \%)$. False negative outcomes may happen due to PCR pack disappointment, or due to either issues with the example or issues playing out the test. Bogus positive outcomes are probably going to be rare $[10,11]$.

One examination in China found that CT checks demonstrated ground-glass opacities in $56 \%$, yet $18 \%$ had no radiological findings. Bilateral and fringe ground glass opacities are the most run of the mill CT discoveries, however they are non-specific. Consolidation, straight opacities and opposite corona sign are other radiological findings. Initially, the sores are kept to one lung, yet as the sickness advances, signs show in the two lungs in $88 \%$ of supposed "late patients" in the investigation gathering (the subset for whom time between beginning of manifestations and chest CT was $6-12$ days). Ground glass opacities are additionally a typical component in kids' disease [12].
Prevention

Because a vaccine in opposition to SARS-CoV-2 is not anticipated to grow to be available till 2021 at the earliest, a key part of handling the COVID-19 pandemic is attempting to decrease the epidemic peak, referred to as knocking down the epidemic curve through numerous measures searching for to lessen the charge of new infections. Slowing the infection charge facilitates decrease the hazard of health offerings being overwhelmed, taking into consideration better remedy of contemporary cases, and provides more time for a vaccine and treatment to be developed [13].

Preventive measures to lessen the chances of contamination in locations with a scourge of the ailment are much like those published for other coronaviruses: live home, keep away from journey and public activities, wash arms with soap and heat water regularly and for as a minimum 20 seconds (proper hand hygiene), practice appropriate respiratory hygiene and keep away from touching the eyes, nose, or mouth with unwashed palms. The CDC recommends masking up the mouth and nostril with a tissue for the duration of any cough or sneeze and coughing or sneezing into the interior of the elbow if no tissue is available. They additionally recommend proper hand hygiene after any cough or sneeze. Social distancing techniques aim to lessen contact of infected men and women with massive organizations by way of closing colleges and workplaces, restricting tour, and canceling mass gatherings [14].

According to the WHO, using masks is handiest recommended if a person is coughing or sneezing or whilst one is taking care of a person with a suspected contamination $[1,2]$.

\section{Management}

To prevent transmission of the virus, the Centers for Disease Control and Prevention (CDC) within the United States recommends that infected individuals stay home except to get clinical care, call in advance before travelling a healthcare provider, put on a face mask while uncovered to an individual or place of a suspected contamination, cover coughs and sneezes with a tissue, often wash palms with soap and water and avoid sharing personal household items. CDC also recommends that individuals wash arms regularly with cleaning soap and water for at the least 20 seconds, specifically after going to the toilet or when hands are visibly dirty, earlier than consuming and after blowing one's nostril, coughing, or sneezing. It in addition endorsed using an alcohol-primarily based hand sanitizer with as a minimum $60 \%$ alcohol, but handiest whilst soap and water are not without problems available. For remote regions where industrial hand sanitizers aren't without difficulty available, WHO advised formulations for the neighborhood production. In both of these formulations the antimicrobial pastime of ethanol or isopropanol is enhanced with the aid of low awareness of hydrogen peroxide even as glycerol acts as a humectant. The WHO advises individuals to keep away from touching the eyes, nose, or mouth 
with unwashed fingers. Spitting in public locations also ought to be avoided $[15,16]$.

Individuals are made do with steady consideration which may incorporate liquid, oxygen backing, and supporting other influenced essential organs. The WHO and Chinese National Health Commission have distributed suggestions for dealing with individuals who are hospitalized with COVID-19. Steroids, for example, methylprednisolone are not prescribed except if the infection is confused by intense respiratory misery syndrome. Intensivists and pulmonologists in the US have aggregated treatment proposals from different organizations into a free asset, the IBCC.CDC suggests that the individuals who presume they convey the infection wear a straightforward face mask [17].

The WHO doesn't prescribe against the utilization of ibuprofen or related non-steroidal mitigating drugs for the treatment of Covid-19 symptoms. Nevertheless, paracetamol is suggested for firstline use [18].

Extracorporeal film oxygenation (ECMO) has been utilized to address the issue of respiratory disappointment, however its advantages are still under consideration.

\section{Individual defensive gear}

The board of individuals contaminated by the infection incorporates playing it safe while applying remedial moves, particularly when performing methods like intubation or hand ventilation that can produce aerosols [19].

CDC plots the particular individual defensive gear and the request in which human services suppliers should put it on when managing somebody who may have COVID-19: 1) outfit, 2) cover or respirator,3) goggles or a face shield, 4) gloves [20].

\section{Mechanical ventilation}

Most instances of COVID-19 are not serious enough to require mechanical ventilation (counterfeit help to help breathing), yet a level of cases do. This is generally regular in more seasoned grownups (those more established than 60 years and particularly those more seasoned than 80 years). Many created nations need more clinic beds per capita, which confines a wellbeing framework's ability to deal with an unexpected spike in the quantity of COVID-19 cases sufficiently serious to require hospitalization. This restricted limit is a huge driver of the need to smooth the bend (to keep the speed at which new cases happen and along these lines the quantity of individuals wiped out at one point in time lower). One concentrate in China discovered $5 \%$ were admitted to escalated care units, $2.3 \%$ required mechanical help of ventilation, and $1.4 \%$ died [21].

\section{Treatment [22-25]}

No prescription are affirmed to treat the malady by the WHO albeit some are suggested by singular national clinical authorities. Research into potential medicines began in January 2020 and a few antiviral medications are in clinical trials. Although new drugs may take until 2021 to develop, a few of the meds being tried are as of now endorsed for different uses or are as of now in cutting edge testing.

Antiviral medicine might be attempted in individuals with extreme disease. The WHO suggested volunteers participate in preliminaries of the viability and wellbeing of potential treatments.

\section{Data innovation}

In February 2020, China propelled a portable application to manage the illness outbreak. Users are approached to enter their name and ID number. The application can distinguish 'close contact' utilizing reconnaissance information and consequently a potential danger of contamination. Each client can likewise check the status of three different clients. In the event that a potential hazard is identified, the application not just suggests self-isolate, it additionally alarms neighborhood wellbeing officials.

In March 2020, the Israeli government empowered security offices to follow cell phone information of individuals expected to have coronavirus. The measure was taken to authorize isolate and ensure the individuals who may come into contact with tainted citizens.

In March 2020, Deutsche Telekom imparted private cellphone information to the government organization, Robert Koch Institute, so as to look into and forestall the spread of the virus.

\section{Mental help}

Tainted people may encounter trouble from isolate, travel limitations, symptoms of treatment, or dread of the contamination itself. To address these worries, the National Health Commission of China distributed a national rule for mental emergency intercession on 27 January 2020.

\section{Prognosis [26-28]}

The severity of COVID-19 varies. The disorder may additionally take a mild direction with few or no signs, corresponding to other not unusual upper respiratory diseases together with the commonplace cold. Mild instances typically recover within two weeks, at the same time as those with severe or essential sickness might also take three to six weeks to get better. Among those who've died, the time from symptom onset to loss of life has ranged from to 8 weeks.

Children of every age are liable to the ailment, but are possibly to have milder signs and symptoms and a far lower risk of extreme 
disorder than adults; in those younger than 50 years, the danger of dying is much less than $0.5 \%$, even as in the ones older than 70 it is more than $8 \%$. Pregnant girls are at particular danger for extreme contamination.

In a few patients COVID-19 may affect the lungs inflicting pneumonia. In the ones maximum critically affected, COVID-19 may unexpectedly progress to acute respiration distress syndrome (ARDS) inflicting breathing failure, septic shock, or multi-organ failure. Complications associated with COVID-19 encompass sepsis, extraordinary clotting, and harm to the heart, kidneys, and liver. Clotting abnormalities, particularly an boom in prothrombin time, have been described in $6 \%$ of those admitted to sanatorium with COVID-19, whilst odd kidney feature is seen in $4 \%$ of this group. Liver injury as shown by using blood markers of liver damage is regularly visible in severe instances.

Many of folks that die of COVID-19 have preexisting conditions, which includes hypertension, diabetes mellitus, and cardiovascular disease. The Italian Istituto Superiore di Sanità suggested that, out of over 2000 deaths from the disease inside the country, $99.8 \%$ had at least one preexisting condition with the common affected person having 2.7. According to the same report, the median time among onset of signs and symptoms and death was eight days with a distinction of one day between sufferers who have been [clarification needed] dealt with in an ICU compared to people who were not. In a observe of early instances, the median time from exhibiting initial symptoms to loss of life become 14 days, with a full range of six to forty one days. In a observe by the National Health Commission (NHC) of China, men had a dying price of $2.8 \%$ at the same time as girls had a death price of $1.7 \%$. Histopathological examinations of post-mortem lung samples show diffuse alveolar damage with cell fibromyxoid exudates in each lungs. Viral cytopathic changes were observed within the pneumocytes. The lung photograph resembled acute respiration misery syndrome (ARDS). In $11.8 \%$ of the deaths pronounced by means of the National Health Commission of China, heart damage became stated by improved levels of troponin or cardiac arrest [28,29].

Availability of medical resources and the socioeconomics of an area may also have an effect on mortality. Estimates of the mortality from the circumstance vary because of those local differences, but also due to methodological difficulties. The under-counting of mild instances can cause the mortality rate to be overestimated. However, the time lag in death happening can suggest the mortality price is underestimated.

It is unknown if past infection provides powerful and long-term immunity in folks who recover from the disorder. Immunity is probable, based on the behaviour of different coronaviruses, but cases in which recovery from COVID-19 were observed via nice checks for coronavirus at a later date have been reported. It is un- clear if these instances are the end result of reinfection, relapse, or testing error.

Concerns have been raised approximately long-time period sequelae of the disorder. The Hong Kong Hospital Authority discovered a drop of $20 \%$ to $30 \%$ in lung capability in some folks that recovered from the sickness, and lung scans advised organ damage.

\section{Epidemiology [30-35]}

The case fatality price (CFR) relies upon on the availability of healthcare, the standard age and health problems in the population and the variety of undiagnosed cases. Preliminary studies has yielded case fatality price numbers among $2 \%$ and $3 \%$; in January 2020 the WHO cautioned that the case fatality charge was about 3\% and 2\% in February 2020 in Hubei. Other CFR numbers, which alter for variations in time of confirmation, demise or remission however are not peer reviewed, are respectively $7 \%$ and 33\% for human beings in Wuhan 31 January. An unreviewed preprint of 55 deaths stated that early estimates of mortality can be too high as asymptomatic infections are missed. They envisioned a mean contamination fatality ratio (IFR, the mortality among infected) starting from $0.8 \%$ to $0.9 \%$. The outbreak in $2019-2020$ has caused at the least 244,517 edit confirmed infections and 10,030 edit deaths.

An observational study of nine people found no vertical transmission from mom to the newborn. Also, a descriptive take a look at in Wuhan located no evidence of viral transmission via vaginal sex (from female to partner), but authors notice that transmission at some stage in sex might arise via different routes.

\section{Phrasing}

The World Health Organization reported on 11 February 2020 that "COVID-19" would be the official name of the ailment. World Health Organization boss Tedros Adhanom Ghebreyesus said "co" means "crown", "vi" for "infection" and "d" for "sickness", while "19" was for the year, as the episode was first distinguished on 31 December 2019. Tedros said the name had been decided to keep away from references to a particular land area (for example China), creature species, or gathering of individuals in accordance with worldwide proposals for naming planned for forestalling stigmatisation.

While the illness is named COVID-19, the infection that causes it is named serious intense respiratory disorder coronavirus 2 or SARS-CoV-2. The infection was at first alluded to as the 2019 novel coronavirus or 2019-nCoV. The WHO moreover utilizes "the COVID-19 infection" and "the infection liable for COVID-19" in open communications.

\section{Research [35-40]}

Due to its key job in the transmission and movement of the infection, ACE2 has been the focal point of a critical extent of research and different remedial methodologies have been suggested. 


\section{Immunization}

There is no accessible immunization, yet investigation into building up an antibody has been embraced by different organizations. Past work on SARS-CoV is being used on the grounds that SARS-CoV-2 and SARS-CoV both utilize the ACE2 receptor to enter human cells. There are three immunization procedures being researched. To begin with, specialists intend to construct an entire infection antibody. The utilization of such an infection, be it inert or dead, means to evoke a brief insusceptible reaction of the human body to another contamination with COVID-19. A subsequent technique, subunit immunizations, plans to make an antibody that sharpens the safe framework to specific subunits of the infection. On account of SARS-CoV-2 such research centers around the S-spike protein that enables the infection to barge in the ACE2 chemical receptor. A third system is the nucleic corrosive antibodies (DNA or RNA immunizations, a novel procedure for making an inoculation). Test immunizations from any of these procedures would need to be tried for security and efficacy.

On 16 March 2020, the principal clinical preliminary of an immunization began with four volunteers in Seattle. The immunization contains an innocuous hereditary code replicated from the infection that causes the disease.

\section{Antivirals}

A few existing antiviral drugs are being taken a gander at to treat COVID-19 and some are moving into clinical trials.

There is conditional proof for remdesivir as of March 2020. Remdesivir represses SARS-CoV-2 in vitro. Phase 3 clinical preliminaries are being directed in the US, in China, and in Italy.

Chloroquine, recently used to treat jungle fever, was being concentrated in China in February 2020, with constructive starter results. Chloroquine and hydroxychloroquine successfully restrain SARS-CoV-2 in vitro, with hydroxychloroquine ending up being more powerful than chloroquine and with an increasingly decent wellbeing profile. Preliminary outcomes from a preliminary proposed that chloroquine is compelling and safe in treating COVID-19 related pneumonia, "improving lung imaging discoveries, advancing an infection antagonistic transformation and shortening the malady course". However, there are calls for more audit of the exploration to date. The Guangdong Provincial Department of Science and Technology and the Guangdong Provincial Health and Health Commission gave a report expressing that chloroquine phosphate "improves the achievement pace of treatment and abbreviates the length of patient's medical clinic remain" and suggested it for individuals determined to have mellow, moderate and extreme instances of novel coronavirus pneumonia. On 17 March, the Italian Pharmaceutical Agency remembered chloroquine and hydroxychloroquine for the rundown of medications with positive fundamental outcomes for treatment of COVID-19. Korean and Chinese Health Authorities prescribe the utilization of chloroquine.

The Chinese seventh release rules additionally incorporate interferon, ribavirin, or umifenovir for use against COVID-19. Teicoplanin seems to hinder SARS-CoV-2 and the related MERS coronaviruses and is seen as a potential treatment for COVID-19. In 2020, a randomized controlled preliminary distributed in the New England Journal of Medicine found that lopinavir/ritonavir was ineffectual in the treatment of serious ailment brought about by SARS-CoV-2 and didn't prompt shorter clinic stays or better results contrasted with standard consideration alone. Nitazoxanide has been suggested for additional in vivo examination in the wake of showing low focus hindrance of SARS-CoV-2.

Studies have exhibited that underlying spike protein preparing by transmembrane protease serine 2 (TMPRSS2) is basic for passage of SARS-CoV-2 by means of association with the ACE2 receptor. These discoveries recommend that the TMPRSS2 inhibitor camostat affirmed for use in Japan for repressing fibrosis in liver and kidney ailment may comprise a viable off-mark treatment

\section{Hostile to cytokine storm}

Tocilizumab has been remembered for treatment rules by China's National Health Commission after a little report was completed. It is experiencing a stage 2 non randomized test at the national level in Italy subsequent to demonstrating constructive outcomes in individuals with extreme disease. Combined with a serum ferritin blood test to recognize cytokine storms, it is intended to counter such improvements, which are believed to be the reason for death in some influenced people. The interleukin- 6 receptor opponent was endorsed by the FDA for treatment against cytokine discharge disorder instigated by an alternate reason, CAR T cell treatment, in 2017.

\section{Inactive neutralizer treatment}

Utilizing blood gifts from individuals who have recouped from COVID-19 is being investigated, a procedure that was pursued for SARS. The instrument of activity is that the antibodies delivered by the insusceptible frameworks of the individuals who have just recuperated are moved to individuals needing them by means of a nonvaccine type of immunization. Other types of latent immunizer treatment, for example, with made monoclonal antibodies, are in development. Convalescent serum creation could be expanded for speedier deployment.

\section{Bibliography}

1. National Health Commission (2020).

2. Diao Y., et al. "Estimating the cure rate and case fatality rate of the ongoing epidemic COVID-19". Med Rxiv (2020). 
3. "2019-nCoV: preliminary estimates of the confirmed-casefatality-ratio and infection-fatality-ratio, and initial pandemic risk assessment". institutefordiseasemodeling.github.io (2020).

4. “Report 4: Severity of 2019-novel coronavirus (nCoV)” (2020).

5. Chen H., et al. "Clinical characteristics and intrauterine vertical transmission potential of COVID-19 infection in nine pregnant women: a retrospective review of medical records". Lancet 395 (2020): 809-815.

6. Cui P., et al. "Clinical features and sexual transmission potential of SARS-CoV-2 infected female patients: a descriptive study in Wuhan, China". Med Rxiv (2020).

7. "Total confirmed cases of COVID-19 per million people". Our World in Data (2020).

8. "Total confirmed deaths due to COVID-19 per million people". Our World in Data (2020).

9. "Novel coronavirus named 'Covid-19': WHO". TODAY online (2020).

10. "The coronavirus spreads racism against - and among - ethnic Chinese". The Economist (2020).

11. "Naming the coronavirus disease (COVID-19) and the virus that causes it". World Health Organization (WHO) (2020).

12. "Novel Coronavirus(2019-nCoV) Situation Report-10". World Health Organization (WHO) 30 (2020).

13. Cascella M., et al. "Features, Evaluation and Treatment Coronavirus (COVID-19)”. Stat Pearls (2020).

14. Chen WH., et al. "The SARS-CoV-2 Vaccine Pipeline: an Overview". Current Tropical Medicine Reports (2020).

15. Roberts M. "Coronavirus: US volunteers test first vaccine". BBC News (2020).

16. Ko WC., et al. "Arguments in favor of remdesivir for treating SARS-CoV-2 infections". International Journal of Antimicrobial Agents (2020): 105933.

17. Wang M., et al. "Remdesivir and chloroquine effectively inhibit the recently emerged novel coronavirus (2019-nCoV) In vitro". Cell Research 30.3 (2020): 269-271.

18. Beeching NJ., et al. "BMJ Best Practices: COVID-19". BMJ (2020).
19. "AIFA e Gilead annunciano che l'Italia è tra i Paesi che testeranno l'antivirale remdesivir per il trattamento del COVID-19". aifa.gov.it (2020).

20. Gao J., et al. "Breakthrough: Chloroquine phosphate has shown apparent efficacy in treatment of COVID-19 associated pneumonia in clinical studies". Bioscience Trends 14.1 (2020): 7273.

21. Yao X., et al. "In Vitro Antiviral Activity and Projection of Optimized Dosing Design of Hydroxychloroquine for the Treatment of Severe Acute Respiratory Syndrome Coronavirus 2 (SARS-CoV-2)". Clinical Infectious Diseases (2020).

22. Touret $\mathrm{F}$ and de Lamballerie X. "Of chloroquine and COVID-19". Antiviral Research 177 (2020): 104762.

23. Multicenter collaboration group of Department of Science Technology of Guangdong Province Health Commission of Guangdong Province for chloroquine in the treatment of novel coronavirus pneumonia (February 2020). "[Expert consensus on chloroquine phosphate for the treatment of novel coronavirus pneumonia]". Zhonghua Jie He He Hu Xi Za 43.3 (2020): 185-188.

24. "Azioni intraprese per favorire la ricerca e l'accesso ai nuovi farmaci per il trattamento del COVID-19". (2020).

25. "Physicians work out treatment guidelines for coronavirus" (2020).

26. "Novel Coronavirus Pneumonia Diagnosis and Treatment Plan (Provisional $7^{\text {th }}$ Edition)". China Law Translate (2020).

27. Baron SA., et al. "Teicoplanin: an alternative drug for the treatment of coronavirus COVID-19?". International Journal of Antimicrobial Agents (2020): 105944.

28. Cao B., et al. "A Trial of Lopinavir-Ritonavir in Adults Hospitalized with Severe Covid-19". The New England Journal of Medicine (2020).

29. Hoffmann M., et al. "The novel coronavirus 2019 (2019-nCoV) uses the SARS-coronavirus receptor ACE2 and the cellular protease TMPRSS2 for entry into target cells". Bio Rxiv (2020).

30. Iwata-Yoshikawa N., et al. "TMPRSS2 Contributes to Virus Spread and Immunopathology in the Airways of Murine Models after Coronavirus Infection". Journal of Virology 93.6 (2019).

31. Liu R and Miller J. "China approves use of Roche drug in battle against coronavirus complications" (2020). 
32. "Effective Treatment of Severe COVID-19 Patients with Tocilizumab". ChinaXiv.org (2020).

33. "3 patients get better on arthritis drug" (2020).

34. "Coronavirus, via libera dell'Aifa al farmaco anti-artrite efficace su 3 pazienti e a un antivirale: test in 5 centri" [Coronavirus, Aifa gives go-ahead to effective anti-arthritis drug on 3 patients and an antiviral: test in 5 centers]. Il Messaggero (in Italian) (2020).

35. "How doctors can potentially significantly reduce the number of deaths from Covid-19". Vox (2020).

36. Ruan Q., et al. "Clinical predictors of mortality due to COVID-19 based on an analysis of data of 150 patients from Wuhan, China". Intensive Care Medicine (2020).

37. Mehta P., et al. "COVID-19: consider cytokine storm syndromes and immunosuppression". The Lancet (2020).

38. "China turns Roche arthritis drug Actemra against COVID-19 in new treatment guidelines". Fierce Pharma (2020).

39. Casadevall A and Pirofski LA. "The convalescent sera option for containing COVID-19". The Journal of Clinical Investigation (2020).

40. Pearce K. "Antibodies from COVID-19 survivors could be used to treat patients, protect those at risk: Infusions of antibodyladen blood have been used with reported success in prior outbreaks, including the SARS epidemic and the 1918 flu pandemic". The Hub at Johns Hopkins University (2020).

\section{Assets from publication with us}

- Prompt Acknowledgement after receiving the article

- Thorough Double blinded peer review

- Rapid Publication

- Issue of Publication Certificate

- High visibility of your Published work

Website: https://www.actascientific.com/

Submit Article: https://www.actascientific.com/submission.php Email us: editor@actascientific.com

Contact us: +919182824667 